\title{
Plasma preptin levels in patients with type 2 diabetes mellitus
}

\author{
Mehmet Kalayci' , (1) Ihsan Halifeoglu2 ${ }^{2}$, (i) Hatice Kalayci' ${ }^{1}$, Kader Ugur ${ }^{3}$, (1) Musa Yilmaz
}

'Department of Medical Biochemistry, Elazig Fethi Sekin City Hospital, Elazig, Turkey

${ }^{2}$ Department of Medical Biochemistry, Firat University, Faculty of Medicine, Elazig, Turkey

${ }^{3}$ Department of Endocrinology, Firat University, Faculty Of Medicine, Elazig, Turkey

${ }^{4}$ Department of Medical Biochemistry, Hitit University, Faculty of Medicine, Corum, Turkey

\begin{abstract}
Objectives: Diabetes mellitus (DM) is a chronic disease characterized by hyperglycemia, and the prevalence is rapidly increasing worldwide. Preptin is a novel molecule with a metabolic effect that is secreted from pancreatic beta cells along with insulin. There are currently few studies about preptin in patients with type 2 DM (T2DM). This study was designed to observe differences in the plasma preptin level in patients with T2DM in comparison with a control group. Methods: The study included 82 patients with T2DM (mean age: $49.00 \pm 5.97$ years; 42 females, 40 males) and 65 healthy individuals (mean age: $41.98 \pm 5.62$ years; 31 females, 34 males). The patients with T2DM were divided into 2 groups: those newly diagnosed and those previously diagnosed and in treatment. The previously diagnosed group was further separated into 2 groups based on their glycated hemoglobin (HbA1c) level: a well-controlled DM (HbA1C value $<7 \%$ ) group and a poorly controlled DM ( $\mathrm{HbA} 1 \mathrm{C}$ value $>7 \%)$ group. The chemistry parameters were analyzed with an Advia 1800 analyzer (Siemens Healthineers, Erlangen, Germany), and hormonal parameters were analyzed with an Immulite 2000 analyzer (Siemens Healthineers, Erlangen, Germany). Plasma preptin levels were measured using the enzymelinked immunosorbent assay method.

Results: Patients with newly or previously diagnosed T2DM had significantly higher glucose, HbA1c, insulin, homeostatic model assessment of insulin resistance (HOMA-IR), total cholesterol, triglyceride, low-density lipoprotein cholesterol, and preptin values ( $p<0.001$ for each), and significantly lower high-density lipoprotein cholesterol levels compared with the control group $(p<0.001)$. The preptin level demonstrated a positive correlation with insulin, HOMA-IR, $\mathrm{HbA1c}$, and glucose levels (for control group: $r=0.452-p=0.000, r=0.472-p=0.000, r=0.389-p=0.001, r=0.286-p=0.021$, respectively; for T2DM group: $r=0.484-p=0.000, r=0.544-p=0.000, r=0.288-p=0.009, r=0.236-p=0.033$, respectively).

Conclusion: The observed correlation between preptin level and DM a suggests that this peptide may have a possible role in the pathogenesis of DM, which may be particularly valuable, given that DM is a major public health problem.

Keywords: Diabetes mellitus, insulin resistance, preptin
\end{abstract}

$D$ abetes mellitus (DM) is a chronic disease characterized by hyperglycemia, the prevalence of which is rapidly increasing across the globe. An inadequacy or deficiency of insulin or the development of insulin resistance in the presence of adequate insulin secretion is the primary root of the pathogenesis. According to International Diabetes Federation data, there were some 425 million patients with diabetes worldwide in 2017, and this number is estimated to reach 629 million by 2045 [1-3]. As the numbers suggest, diabetes is considered a major public health issue.
Preptin was first discovered in 2001 in rat experiments. It is a peptide hormone consisting of 34 amino acids which is secreted along with insulin from the pancreatic beta cells $[4,5]$. As an endocrine peptide, preptin is thought to activate the insulinlike growth factor receptor 2 (IGF2R), and as a result, induces calcium-dependent insulin secretion in association with protein $C$ and phospholipase $C$ when the glucose concentration is high [6]. In addition, preptin has insulin-like effects on bone metabolism, such as boosting cellular differentiation and the affecting the functions of osteoblasts and osteoclasts [7].

Address for correspondence: Mehmet Kalayci, MD. Department of Medical Biochemistry, Elazig Fethi Sekin City Hospital, Elazig, Turkey Phone: +90 5541182255 E-mail: dr_mehmetkalayci@msn.com ORCID: 0000-0001-9122-9289

Submitted Date: August 16, 2018 Accepted Date: October 08, 2018 Available Online Date: January 14, 2019

${ }^{\circ}$ Copyright 2018 by International Journal of Medical Biochemistry - Available online at www.internationalbiochemistry.com OPEN ACCESS This work is licensed under a Creative Commons Attribution-NonCommercial 4.0 International License. 
Preptin plays a role in metabolic pathways. There is currently a very limited number of studies regarding preptin in patients with T2DM. The aim of the present study was to investigate how the plasma preptin level was different in patients with T2DM compared with healthy controls.

\section{Materials and Methods}

The study included 82 patients with T2DM (42 females and 40 males) from the endocrinology outpatient clinic of Firat University Hospital, along with 65 healthy control participants (31 females, 34 males). The diagnosis of T2DM was based on the American Diabetes Association criteria [1].

Patients with T2DM were categorized in 2 groups: those recently diagnosed with DM and those previously diagnosed and in treatment. The group of patients who had been previously diagnosed was further stratified according to glycated hemoglobin $(\mathrm{HbA} 1 \mathrm{c})$ level as well-controlled DM ( $\mathrm{HbA} 1 \mathrm{c}$ $<7.0 \%$ ) and poorly controlled DM (HbA1c >7.0\%) [8]. There were 22 patients in the newly diagnosed DM group and 60 patients in the previously diagnosed/in treatment group. Among the previously diagnosed patients, 23 had good glycemic control and 37 had poor glycemic control. Forty-two of the previously diagnosed patients were using oral anti-diabetic agents (OADs), 13 were treated with an OAD+insulin, and 5 were receiving only insulin treatment therapy.

The height, weight, and waist circumference of all of the study participants were measured. Body mass index (BMI) was calculated as body weight divided by the square of height (kg/ $\mathrm{m}^{2}$ ). Insulin resistance was calculated using the homeostatic model assessment of insulin resistance formula: HOMA-IR=[fasting insulin $(\mu \mathrm{U} / \mathrm{mL}) \times$ fasting glucose $(\mathrm{mmol} / \mathrm{L}) / 22.5]$.

This study was approved by the Ethics Committee of Firat University Faculty of Medicine (date 13.05.2011, session \#8). All of the participants were informed about the study protocol and provided written, informed consent.

Blood samples for both groups were obtained in 3 different blood collection tubes following 8 to 12 hours of fasting. One tube contained a serum separator (BD Vacutainer SST II Plus; Becton, Dickenson and Co., Franklin Lakes, NJ, USA), 1 was coated with dipotassium ethylenediaminetetraacetic acid (BD Vacutainer SST II Advance, Becton, Dickenson and Co., Franklin Lakes, NJ, USA), and 1 contained aprotinin (BD Vacutainer BD Vacutainer EDTA Aprotinin Tube; Becton, Dickenson and Co., Franklin Lakes, NJ, USA). The blood samples in serum separator tubes were centrifuged at $4000 \mathrm{rpm}$ for 10 minutes, and the obtained serum was used for fasting glucose and lipid profile (total cholesterol, high-density lipoprotein cholesterol [HDL-C], low-density lipoprotein cholesterol [LDL-C], triglyceride) measurement using an Advia 1800 Chemistry System analyzer (Siemens Healthineers, Erlangen, Germany), as well as a serum insulin level measurement using a Siemens Immulite 2000 analyzer (Siemens Healthineers, Erlangen, Germany). HbA1c concentrations were measured using a Shimadzu Prominence
Modular HPLC analyzer (Shimadzu Corp., Kyoto, Japan) and the ImmuChrom brand commercial kit (ImmuChrom GmbH, Heppenheim, Germany). Blood samples drawn into tubes containing aprotinin were centrifuged at $4000 \mathrm{rpm}$ for 10 minutes, and the obtained plasma was aliquoted to small volume tubes and stored at $-20^{\circ} \mathrm{C}$ until performing the preptin analysis.

The plasma preptin level was measured using the Human Preptin ELISA kit (USCN Life Science, Inc., Wuhan, China) in accordance with the manufacturer's instructions and a Triturus Enzyme Immunoassay Analyzer (Grifols, S.A., Barcelona, Spain). The minimum detection limit of preptin was $62.5 \mathrm{pg} /$ $\mathrm{mL}$. The intra-assay and inter-assay coefficients of variation for plasma preptin were $3.8 \%$ and $10.6 \%$, respectively.

The data were expressed as mean \pm SD. Normality assessment was performed with the Kolmogorov-Smirnov test. A chisquare test was used for nominal parameters. Comparisons between the groups were made using one-way analysis of variance with the Tukey test, and the correlation between parameters within groups was analyzed with Pearson's correlation test. $P<0.05$ was accepted as statistically significant.

\section{Results}

Table 1 summarizes the demographic and biochemical properties of the study groups. There was a statistically significant difference between the control and T2DM groups in the mean age, BMl, and waist circumference $(p<0.001)$. Glucose, HbA1c, insulin, HOMA-IR, total cholesterol, LDL-C, and triglyceride levels were higher ( $p<0.001$ for each), and the HDL-C level was lower $(p<0.001)$ in the T2DM group compared with the control group (Table 1).

The plasma preptin levels were significantly higher in patients with T2DM than in the healthy controls $(437.84 \pm 133.4 \mathrm{pg} / \mathrm{mL}$ vs. $284.77 \pm 55.49 \mathrm{pg} / \mathrm{mL} ; \mathrm{p}<0.001$ ) (Fig. 1).

The preptin level was not statistically significantly different between females and males in the control group (283.67 \pm 59.5 $\mathrm{pg} / \mathrm{mL}$ vs. $285.76 \pm 52.4 \mathrm{pg} / \mathrm{mL} ; \mathrm{p}>0.05$ ) or in the T2DM group (440.31 $\pm 144.6 \mathrm{pg} / \mathrm{mL}$ vs. $435.25 \pm 122.1 \mathrm{pg} / \mathrm{mL} ; \mathrm{p}>0.05)$.

Neither was there a significant difference in the preptin level $(427.45 \pm 124.7 \mathrm{pg} / \mathrm{mL}$ vs. $441.65 \pm 137.2 \mathrm{pg} / \mathrm{mL} ; \mathrm{p}>0.05)$ between the newly diagnosed DM and the previously diagnosed and in treatment T2DM groups.

When the preptin level was analyzed after stratifying patients based on HbA1c level, patients with poorly controlled T2DM had a higher preptin level compared with the patients with well-controlled T2DM $(454.1 \pm 154.7 \mathrm{pg} / \mathrm{mL}$ vs. $421.7 \pm 102.9 \mathrm{pg} /$ $\mathrm{mL})$, but the difference was not statistically significant ( $p>0.05$ ). In the control group, the plasma preptin level revealed a statistically significant positive correlation with insulin $(r=0.452$; $p=0.0001)$, HOMA-IR $(r=0.472 ; p=0.0001), H b A 1 c \quad(r=0.389$; $p=0.001)$, and glucose ( $r=0.286 ; p=0.021)$ levels (Fig. 2). In the T2DM group, the plasma preptin level also had a statistically significant positive correlation with insulin $(r=0.484$; 
Table 1. Demographic and biochemical properties of the control, newly diagnosed T2DM, and previously diagnosed T2DM groups

Previously diagnosed T2DM $n=60$

\begin{tabular}{|c|c|c|c|c|}
\hline & $\begin{array}{l}\text { Control } \\
n=65\end{array}$ & $\begin{array}{l}\text { Newly diagnosed } \\
\text { T2DM } \\
n=22\end{array}$ & $\begin{array}{l}\text { Well-controlled } \\
\text { (HbA1c < 7.0\%) } \\
n=23\end{array}$ & $\begin{array}{l}\text { Poorly controlled } \\
\text { (HbA1c }>7.0 \% \text { ) } \\
n=37\end{array}$ \\
\hline Age (years) & $41.98 \pm 5.62$ & $48.09 \pm 6.9^{b}$ & $50.69 \pm 4.11^{b}$ & $48.48 \pm 6.28^{b}$ \\
\hline BMI $\left(\mathrm{kg} / \mathrm{m}^{2}\right)$ & $26.04 \pm 2.93$ & $29.88 \pm 3.0^{b}$ & $31.44 \pm 4.51^{\mathrm{b}}$ & $31.60 \pm 4.07^{b}$ \\
\hline $\mathrm{HbA1C}(\%)$ & $4.96 \pm 0.23$ & $8.36 \pm 1.62^{b, c}$ & $6.14 \pm 0.59^{b}$ & $9.17 \pm 1.40^{b, c}$ \\
\hline Insulin ( $\mu \mathrm{lU} / \mathrm{mL})$ & $6.30 \pm 1.57$ & $10.15 \pm 5.28^{\mathrm{a}}$ & $10.14 \pm 7.21^{\mathrm{a}}$ & $10.52 \pm 4.69^{b}$ \\
\hline HOMA-IR & $1.45 \pm 0.42$ & $4.93 \pm 3.2^{b, c}$ & $3.64 \pm 2.91^{\mathrm{a}}$ & $5.78 \pm 2.89^{b, c}$ \\
\hline Cholesterol (mg/dL) & $182.06 \pm 22.75$ & $206.82 \pm 27.87^{a}$ & $201.69 \pm 29.98^{\mathrm{a}}$ & $213.11 \pm 36.01^{b}$ \\
\hline HDL-cholesterol (mg/dL) & $49.37 \pm 9.11$ & $39.91 \pm 6.99^{b}$ & $45.17 \pm 7.71$ & $42.16 \pm 9.77^{a}$ \\
\hline Diabetes duration (years) & - & - & $3.87 \pm 3.53$ & $7.62 \pm 4.91$ \\
\hline
\end{tabular}

a: $\mathrm{p}<0.01$; Compared with the control group.

b: $p<0.001$; Compared with the control group.

c: $\mathrm{p}<0.001$; Compared with the well-controlled T2DM group.

$\mathrm{p}<0.05$ statistically significant.

BMI: Body mass index; HbA1C: Glycated hemoglobin; HDL: High-density lipoprotein; HOMA-IR: Homeostatic model assessment of insulin resistance; LDL: Low-density

lipoprotein; T2DM: Type 2 diabetes mellitus.

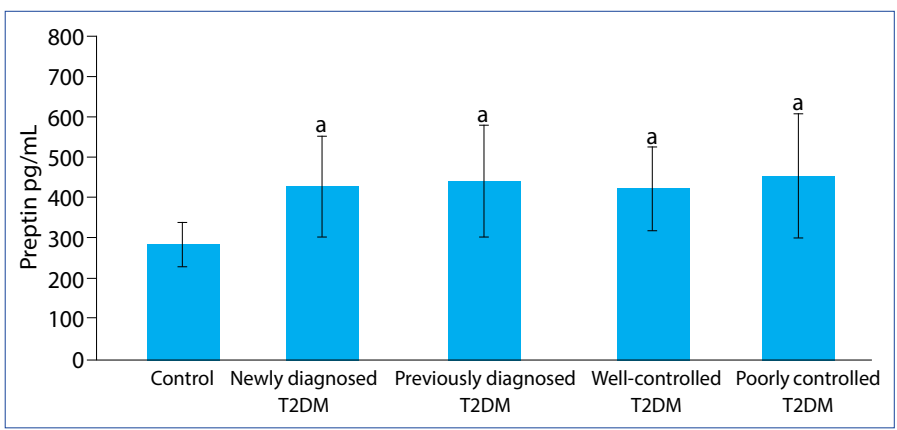

Figure 1. Plasma preptin levels of the control and type 2 diabetic groups (T2DM).

a: $p<0.001$; Compared with the control group.

$p=0.0001)$, HOMA-IR ( $r=0.544 ; p=0.0001), \mathrm{HbA} 1 \mathrm{C} \quad(r=0.288$; $p=0.009)$, and glucose $(r=0.236 ; p=0.033)$ levels (Fig. 3$)$. The preptin level did not show any correlation with age, BMI, waist circumference, or lipid levels in either of the groups.

\section{Discussion}

T2DM is characterized by a disturbance in insulin secretion, the presence of insulin resistance, excessive hepatic glucose production, and abnormal lipid metabolism. In particular, visceral and central obesity are very common in T2DM. The accompanying central or visceral obesity is thought to be a part of the pathogenic process in T2DM $[9,10]$. The fact that higher
BMI values have been observed in diabetic patients supports this hypothesis.

Insulin resistance plays an important role in the pathogenesis of T2DM, and it leads to increased insulin secretion from pancreatic beta cells in order to compensate for elevated blood glucose levels. Insulin resistance causes elevation in plasma glucose concentration as a result of the reduction in utilization of peripheral glucose and the increase in hepatic glucose production [9]. Insulin resistance in the adipose tissue results in increased lipolysis in adipocytes and fatty acid release from adipocytes, increased lipid synthesis in hepatocytes, and reduced insulin sensitivity in liver and muscle tissues [11].

In this study, we investigated the T2DM-dependent change in circulating levels of preptin, a novel peptide secreted from pancreatic beta cells. Patients with T2DM were found to have significantly higher levels of plasma preptin compared with healthy controls. To the best of our knowledge, there is only 1 previous study that examined the change in preptin level in patients with T2DM. In that study, Yang et al. [12] found higher levels of preptin in patients with T2DM compared with a control group. Higher preptin levels have also been reported in studies of patients with gestational DM and polycystic ovary syndrome [13-15]. In our study, after stratifying our diabetic patients according to the target $\mathrm{HbA} 1 \mathrm{c}$ level of $7 \%$, we observed higher preptin levels among patients with poorly controlled diabetes compared with those with controlled diabetes. We believe this increase occurs as a response to the elevated 


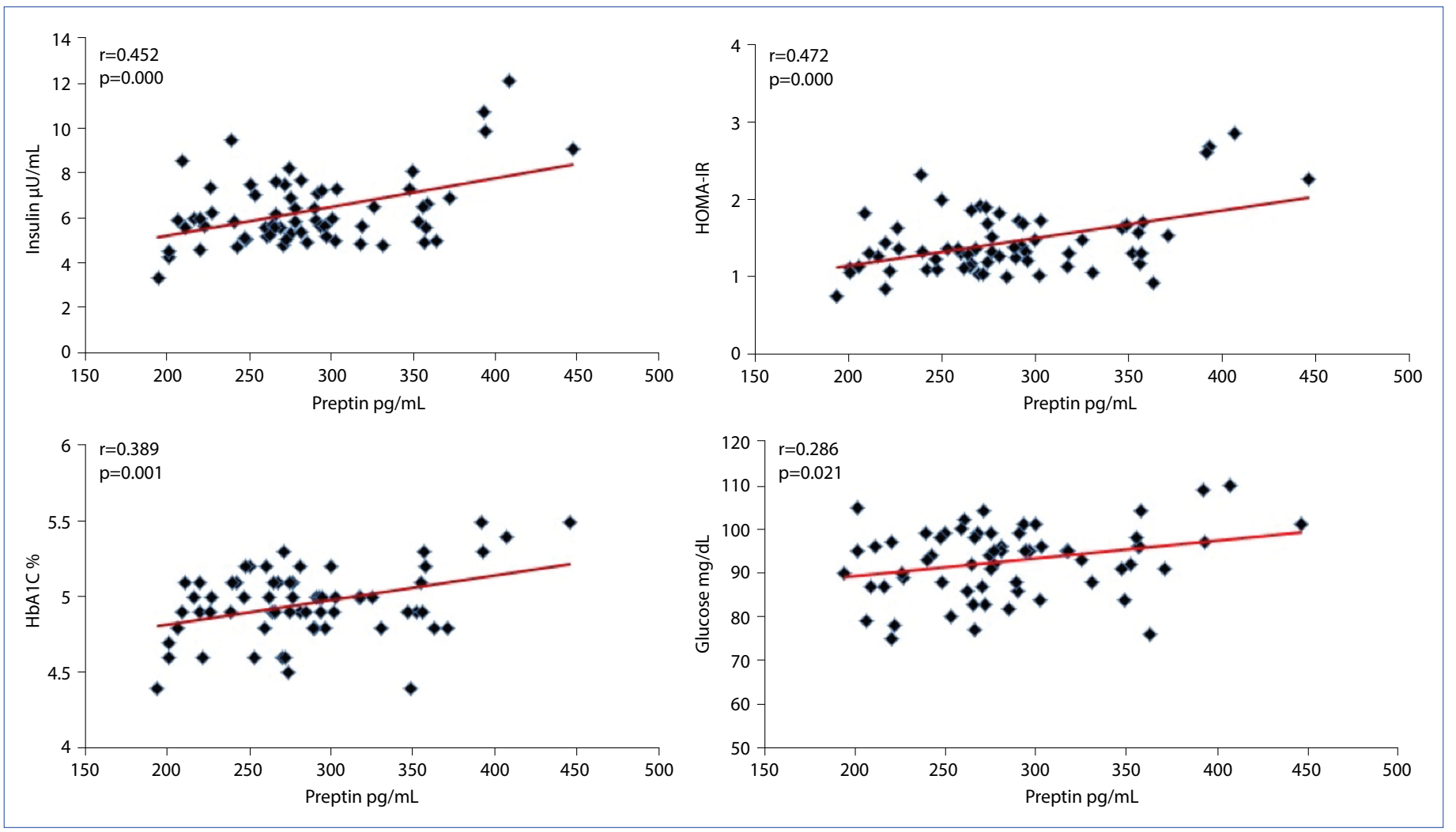

Figure 2. Correlation of preptin level with the parameters of insulin, HOMA-IR, HbA1C, and glucose in the control group.

HbA1C: Glycated hemoglobin; HOMA-IR: Homeostatic model assessment of insulin resistance.

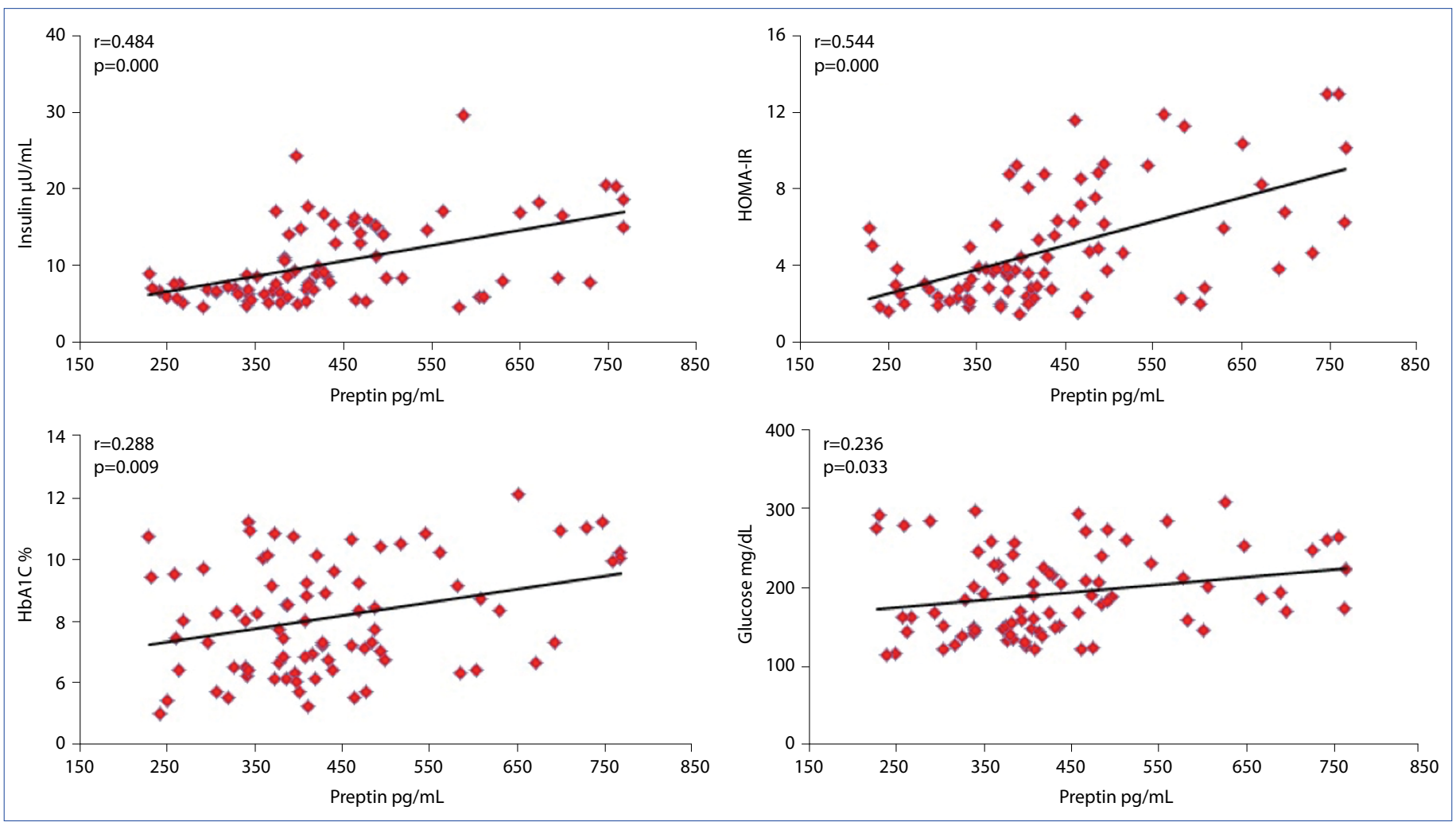

Figure 3. Correlation of preptin level with the parameters of insulin, $\mathrm{HOMA}-\mathrm{IR}, \mathrm{HbA} 1 \mathrm{C}$, and glucose in the T2DM group.

HbA1C: Glycated hemoglobin; HOMA-IR: Homeostatic model assessment of insulin resistance. 
glucose levels. An infusion of preptin to an isolated rat pancreas was shown to increase the second phase of glucose-mediated insulin secretion by $30 \%$, whereas an infusion of anti-preptin antibodies reduced the first phase of insulin secretion by $29 \%$ and the second phase by $26 \%$ [4]. In their 2012 study, Cheng et al. [6] found that an intravenous preptin infusion administered to rats induced a reduction in the plasma glucose level that was related to insulin secretion during glucose loading. They also reported that the effect of preptin on insulin secretion was similar to that of glibenclamide, which stimulates insulin secretion by inhibiting adenosine-5'-triphosphate-responsive potassium channels in pancreatic beta cells [6]. Similar to our research, the preptin level showed a positive correlation with insulin, HOMA$\mathrm{IR}$, glucose, and HbA1c levels in a study conducted by Yang et al.[12]. Studies of patients with gestational DM and polycystic ovary syndrome also found a positive correlation between the preptin level, HOMA-IR, and the insulin level $[13,15]$. Bringing all these data together, we think that preptin is directly related to glucose-mediated insulin secretion. An important question is the degree of the association between preptin concentrations and the insulin that is increased in circulation due to insulin resistance in T2DM. It is still controversial whether the cause of the increased insulin level seen as a result of resistance is due to increased capacity of the pancreatic cells or stimulation of insulin secretion by preptin.

A recent study found that preptin activates the IGF2R, and this finding suggests that activated IGF2R might induce calciumdependent insulin secretion at high glucose concentrations in association with protein kinase $C$ and phospholipase $C$ [6]. The fact that preptin has a similar effect to that of glibenclamide with regard to insulin secretion suggests that preptin analogues may possibly be used in the treatment of T2DM.

\section{Conclusion}

Further studies are needed to understand the complicated signaling pathways as well as physiological significance of preptin, particularly in humans. Our knowledge related to the mechanism of action of preptin and its role in diabetes development continues to grow. The mechanisms mentioned above, and the diabetes-associated changes observed in concentrations of preptin support the idea that this molecule plays an important role in the pathogenesis of T2DM, which is an important public health problem of the present and the future.

\section{Conflict of interest: None declared.}

Ethics Committee Approval: This study was approved by the Ethics Committee of Firat University Faculty of Medicine (date 13.05.2011, session \#8). All of the participants were informed about the study protocol and provided written, informed consent.

Financial Disclosure: This study was supported by Firat University Scientific Research Projects.
Peer-review: Externally peer-reviewed.

Authorship contributions: Concept - M.K., I.H., H.K.; Design M.K., I.H., H.K.; Supervision - M.K., I.H., H.K.; Materials - M.K., H.K., K.U., M.Y.; Data collection \&/or processing - M.K., H.K., K.U., M.Y.; Analysis and/or interpretation - M.K., I.H., H.K., M.Y.; Literature search - M.K., I.H., H.K., M.Y.; Writing - M.K., I.H., H.K., M.Y.; Critical review - M.K., I.H.

\section{References}

1. American Diabetes Association. 2. Classification and Diagnosis of Diabetes: Standards of Medical Care in Diabetes-2018. Diabetes Care 2018;41:S13-27. [CrossRef]

2. International Diabetes Federation. IDF Diabetes Atlas. 8th edition. Brussels: International Diabetes Federation; 2017.

3. Akın S, Erdem ME, Kazan S, Aliustaoğlu M. The relationship between helicobacter pylori infection and glycemic regulation in type 2 diabetic patients. Nobel Med 2014;10:32-5.

4. Buchanan CM, Phillips AR, Cooper GJ. Preptin derived from proinsulin-like growth factor II (prolGF-II) is secreted from pancreatic islet beta-cells and enhances insulin secretion. Biochem J 2001;360:431-9. [CrossRef]

5. Aydin S. Three new players in energy regulation: preptin, adropin and irisin. Peptides 2014;56:94-110. [CrossRef]

6. Cheng KC, Li YX, Asakawa A, Ushikai M, Kato I, Sato Y, et al. Characterization of preptin-induced insulin secretion in pancreatic $\beta$-cells. J Endocrinol 2012;215:43-9. [CrossRef]

7. Ismayilnajadteymurabadi $H$, Konukoglu $D$. The relationship between preptin, Forkhead box protein $\mathrm{O} 1$ and mechanistic target of rapamycin levels in prediabetic patients. J Biol Regul Homeost Agents 2017; 31:399-405.

8. American Diabetes Association. Glycemic Targets: Standards of Medical Care in Diabetes-2018. Diabetes Care 2018;41:S55-64.

9. Powers AC. Diabetes Mellitus. In: Longo DL, Kasper DL, Jameson $\mathrm{JL}$, Fauci AS, Hauser SL, Loscalzo J, editors. Harrison's Principles of Internal Medicine. 18th edition USA: McGraw-Hill; 2012.

10. Lee JJ, Beretvas SN, Freeland-Graves JH. Abdominal Adiposity Distribution in Diabetic/Prediabetic and Nondiabetic Populations: A Meta-Analysis. J Obes 2014;2014:697264. [CrossRef]

11. Lee MJ, Wu Y, Fried SK. Adipose tissue heterogeneity: Implication of depot differences in adipose tissue for obesity complications. Mol Aspects Med 2013;34:1-11. [CrossRef]

12. Yang G, Li L, Chen W, Liu H, Boden G, Li K. Circulating preptin levels in normal, impaired glucose tolerance, and type 2 diabetic subjects. Ann Med 2009;41:52-6. [CrossRef]

13. Aslan M, Celik O, Karsavuran N, Celik N, Dogan DG, Botan E, et al. Maternal serum and cord blood preptin levels in gestational diabetes mellitus. J Perinatol 2011;31:350-5. [CrossRef]

14. Mierzwicka A, Kuliczkowska-Plaksej J, Kolačkov K, Bolanowski M. Preptin in women with polycystic ovary syndrome. Gynecol Endocrinol 2018;27:1-6. [CrossRef]

15. Celik O, Celik N, Hascalik S, Sahin I, Aydin S, Ozerol E. An appraisal of serum preptin levels in PCOS. Fertil Steril 2011;95:314-6. 